\title{
Arachnoiditis Ossificans Mimicking Spinal Intradural Extramedullary Tumor: A Case Report and Review of the Literature
}

\author{
Yao Christian Hugues Dokponou ${ }^{1,2 *}$ (D) Inas El Kacemi ${ }^{1,2}$, Fernand Nathan Imoumby ${ }^{1,2}$, \\ Franck Loukou Kouakou1,2, Sofia El Akroud1,2, Miloud Gazzaz ${ }^{1,2}$
}

${ }^{1}$ Mohammed V Military Teaching Hospital, Rabat, Morocco

${ }^{2}$ Faculty of Medicine and Pharmacy, Mohammed V University, Rabat, Morocco

Email: *dokponou2407@gmail.com

How to cite this paper: Dokponou, Y.C.H., El Kacemi, I., Imoumby, F.N., Kouakou, F.L., El Akroud, S. and Gazzaz, M. (2021) Arachnoiditis Ossificans Mimicking Spinal Intradural Extramedullary Tumor: A Case Report and Review of the Literature. Open Journal of Modern Neurosurgery, 11, 157-163.

https://doi.org/10.4236/ojmn.2021.113019

Received: April 30, 2021

Accepted: June 29, 2021

Published: July 2, 2021

Copyright $\odot 2021$ by author(s) and Scientific Research Publishing Inc. This work is licensed under the Creative Commons Attribution International License (CC BY 4.0).

http://creativecommons.org/licenses/by/4.0/

\begin{abstract}
Arachnoiditis ossificans is an intradural extramedullary lesion resulting from an unusual chronic meningeal inflammatory process and it is thought to be the sequela of end-stage adhesive arachnoiditis secondary to surgery, trauma, arachnoid hemorrhage, meningeal irritation, myelography (particularly oil-based contrast agents), and spinal anesthesia. The spinal arachnoiditis ossificans may be silent or cause a variety of symptoms depending on its location and uncommonly happen to cause spinal cord compression. Very little attention has been paid to the management and outcome of this rare condition in the neurosurgical reem. We report the case of a 45 years old man admitted with L1 - L2 arachnoiditis ossificans revealed by polyradiculopathy on incomplete cauda equina syndrome mimicking spinal canal tumors. The patient underwent surgery and we performed the laminectomy of L1 - L2, total resection of the lesion, followed by a complete remission of the hemiparesis after three months of kinesitherapy nursing.
\end{abstract}

\section{Keywords}

Management, Outcome, Arachnoiditis Ossificans, Spinal Tumor, Case Report

\section{Introduction}

Arachnoiditis ossificans was described in 1971 by Kaufman and Dunsmore, as intradural calcifications in the setting of chronic arachnoiditis. The process starts with dural inflammation and arachnoid membranes that eventually lead to scarring and fibrosis, which can ultimately calcify. This condition is usually as- 
sociated with progressive neurological deficits and the patient usually had a previous medical history of invasive intervention, trauma, surgery, infection, subdural hemorrhage, or myelography. Three (3) types have been described: Type 1: semicircular calcification involving a portion of the dural sac, mostly in the thoracic spine; Type 2: circular calcification involving circumference of the dural sac in the thoracic or lumbar spine; Type 3: calcifications affecting entire contents of the dural sac, crossing the caudal roots in the lumbar spine [1] [2]. CT-Scan and MRI are important for the diagnosis suspicion and to be confirmed by pathological anatomy findings in case the patient undergoes surgery. The main difficulties of the diagnosis are the prolonged course of the medical history, the clinical findings that are usually related to intradural extramedullary tumors without significant past medical history, and the tumor-like lesion found in neuroradiological imaging. All these conditions can easily lead to a missed diagnosis and hardened the operative decision-making. This clinical case we report is not only rare, but it is also unique for the patient's past medical history is unremarkable for all those predisposing factors and his clinical findings were polyradiculopathy on incomplete cauda equina syndrome; the overall mimicking a spinal progressive compressive tumor. We, therefore, find it important to raise scientific awareness about this condition by sharing the positive outcome of our management.

\section{Case Presentation}

A 45-year-old man, with no past medical history, was admitted to the neurosurgery department of the Military Teaching Hospital of Rabat on the $2^{\text {nd }}$ of January 2021 for a polyradiculopathy on incomplete cauda equina syndrome. The patient report 8 years history of dorsal intermittent pain that is lately complicated with a paraparesis and pyramidal syndrome more severe on the right lower limb and characterized by a foot drop, a steppage gait, muscle weakness measured to be $3 / 5$ on the muscle tone scale for the right lower limb. The reflexes were lost on the right lower limb. There were no signs of genital or sphincter impairment. The patient denied any history of arterial hypertension, diabetes, or trauma prior to the appearance of symptoms. He declares no other medical problems. His family history was unremarkable. The patient was alert, in good general status, without fever at the admission.

The laboratory test of the blood sample was normal. The Magnetic Resonance Imaging (MRI) of the lumbar spine revealed an L1, intra-thecal, round, iso-signal intensity lesion on T1- and T2-weighted with homogenous enhancement on T1-Gadolinium, suggesting a schwannoma (Figure 1). Knowing that calcifications on MRI are supposed to be a signal loss on both T1- and T2-weighted images of the dural sac and the nerve roots, we could think of any other tumor lesion but not a calcification.

The patient underwent surgery for the excision of the tumor. After a bilateral T12 - L1 - L2 muscle dissection is performed and the exposure is maintained with the bilateral autostatic retractors, we performed laminectomy of L1 - L2 
and exposed the dura matter which was opened under a surgical microscope and we found an oval shape, white, hard, and tender structure lining on the inner surface of the dura matter and adherent to the cauda equina nerves roots (Figure 2). We proceed with the dissection of this lesion with a total macroscopical excision. The operative finding is then not a schwannoma but an intradural extramedullary calcified round and flat lesion of $11 \times 9 \mathrm{~mm}$ (Figure 3 ). The histopathological analysis of the sample found a calcified tissue surrounded by dense fibrous tissue linked with loose connective tissue, allowing to conclude on an arachnoiditis ossificans (Figure 4).

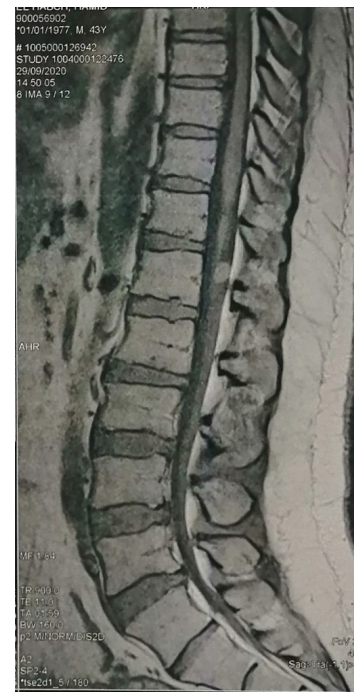

(a)

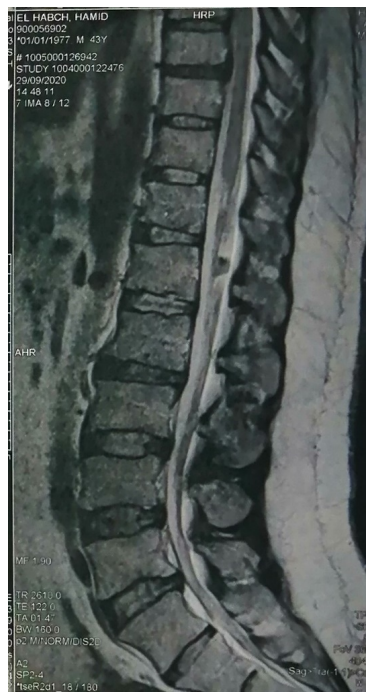

(b)

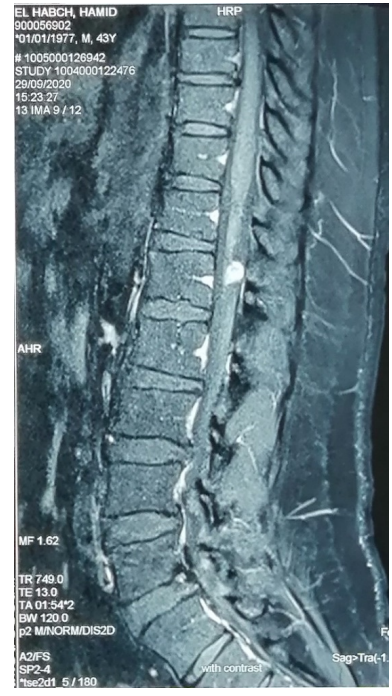

(c)

Figure 1. Sagittal magnetic resonance imaging of the lumbar spine showing the intra thecal arachnoid calcification appearing as an iso-signal intensity linear structure on T1 (a) and T2-weighted (b) (white arrow). (c) The lesion is homogeneously enhanced after gadolinium injection (black arrow).
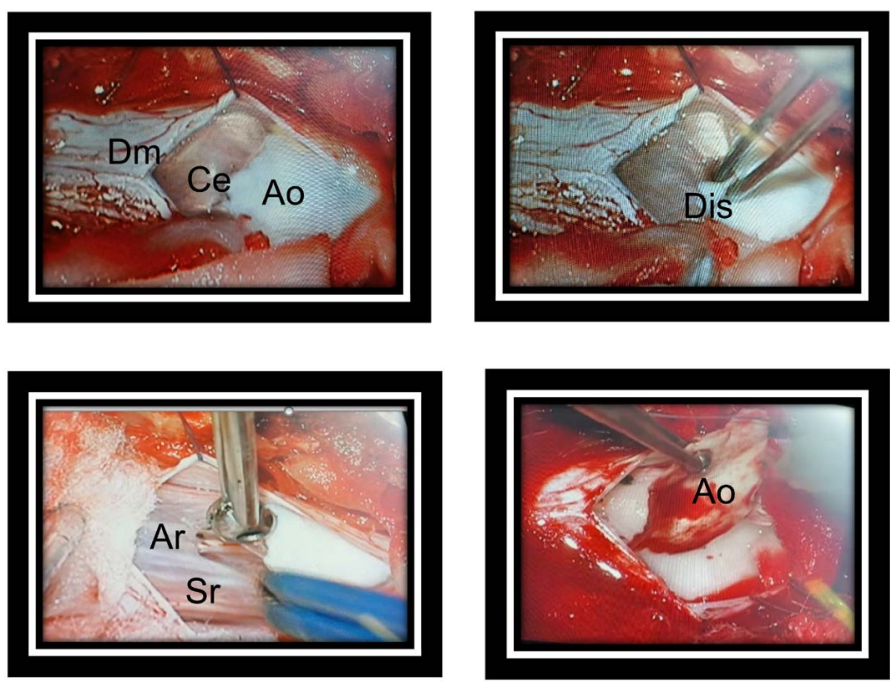

Figure 2. Intra-operative view, $\mathrm{Dm}=$ Dura matter, $\mathrm{Ar}=$ Arachnoid, $\mathrm{Ce}=$ Cauda equina, Ao $=$ Arachnoiditis ossificans, Dis $=$ Dissection, $\mathrm{Sr}=$ Spinal roots. 


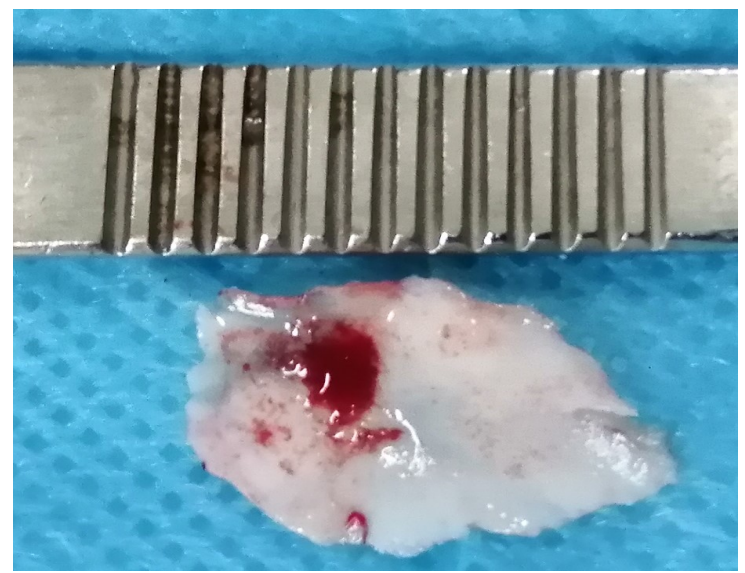

Figure 3. Arachnoiditis ossificans. About 11 millimeters of whitish calcified flattened lesion.

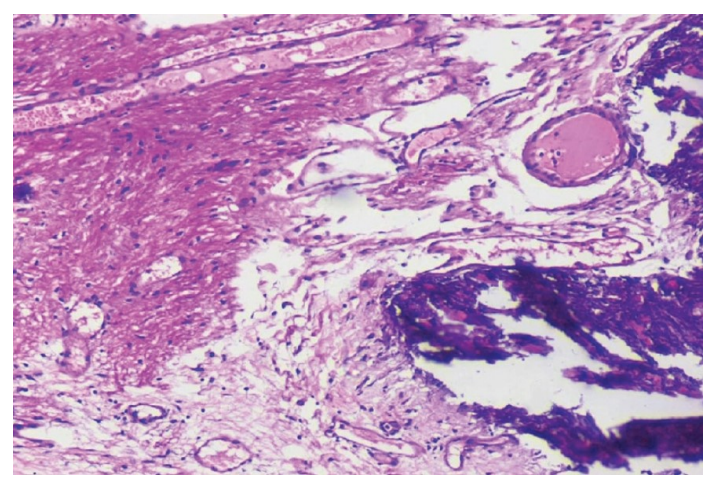

Figure 4. Histopathology, showing calcified tissue with loose connective tissue, related by dense fibrous tissue. Photo $[\mathrm{HE} \times 40$. High power].

The patient did well in the post-operative period with a progressive remission of his signs and symptoms and was discharged 5 days after the surgical procedure. At one and three months follow-up during which the patient underwent kinesitherapy nursing by 20 sessions of functional rehabilitation of the muscles of the lower limbs, he got complete remission of the hemiparesis and he is now able to live a normal life.

\section{Discussion}

The spinal arachnoiditis ossificans is a rare pathology of unknown etiology and its pathogenesis is not yet understood. All we know is the probable chronic inflammatory process of the arachnoidal tissue that will, later on, be fibrosis and calcified. Many predisposing factors have been cited and the majority of published cases have at least one predisposing factor from spinal trauma to surgery with spinal exploration procedures such as myelography (particularly oil-based contrast agents) being the most common. Very few cases, less than ten (10) were reported no having any predisposing factor exactly as the patient of this study. There is no specific clinical presentation and progressive spinal cord compression, polyradiculopathy and cauda equina lesions are rarely reported and more- 
over, this disease affects more females $56 \%$ than males $44 \%$ [3]. Exactly like the clinical findings of our patient of this case report, other authors argue that patients commonly present with chronic low back pain with or without radicular symptoms, which may simulate spinal stenosis and polyneuropathy. Paraesthesia and hypoesthesia are common. Gait disturbance, incontinence, and myelopathic symptoms dominate in a minority of patients [4]. Most past reports indicate arachnoiditis ossificans is generally associated with neurologic deficits, but patients can also be relatively asymptomatic regardless of the degree of ossification [5].

The typical radiological findings are spontaneous hyperdensity of the dural sac on the CT Scan and signal loss of the lesion on the MRI [5] [6] [7] [8]. Meanwhile, in our study, one of the difficulties encountered was that the MRI finding was different and more suggesting of spine schwannoma. On the MRI There was no loss of signal of the L1 lesion but we find a round well-circumscribed lesion presenting as a tissue structure on the MRI. Couple with fact that the clinical presentation of our patient was a polyradiculopathy with incomplete cauda equina syndrome, it was urgent to operate on the patient before he presents signs of genital or sphincter impairment. We took the patient for surgery to operate on a spinal tumor, the schwannoma and find a lesion that was whitish and calcified. This finding is confirmed by the histopathological analysis.

The management of arachnoiditis ossificans is not yet codified and the decision neither to operate nor to undergo surgery or conservative treatment and when and who should be operated on is still not clear. Some authors suggested that the patient should undergo conservative treatment in case he presented mild symptoms. Treatment choices are limited and include intrathecal steroid injection and spinal cord stimulation for pain management. Surgical lysis of adhesions and arachnoid septations is usually not indicated or successful. From the 46 cases of arachnoiditis ossificans reviewed by Archana et al. [3], Thirty-seven underwent thoracic and Lumbar surgery while six of them were a candidate for the conservative treatment based on the severity of their symptoms, their past medical history, and general status.

The outcome depends on the overall treatment choice and the clinical status of the patient prior to surgery.

\section{Conclusion}

The treatment choice of spinal arachnoiditis ossificans is still controversial. Conservative pain management treatment is preferable while dealing with mild neurological symptoms. In case of progressive neurological deterioration, surgery such as laminectomy for dural decompression combined with the resection of the lesion should be thought.

\section{Informed Consent}

The patient gave his informed consent to publish his case. 


\section{Disclosure}

The authors did not receive any funding for the preparation of this case report.

\section{Conflicts of Interest}

The authors declare that they have no conflicts of interest.

\section{Authors' Contributions}

Yao Christian Hugues Dokponou: Conceptualization, writing original draft, \& editing. Inas El KACEMI: Writing, review \& editing. Fernand Nathan IMOUMBY: Writing, review \& editing. Franck Loukou KOUAKOU: Review \& editing. El AKROUD Sofia: Writing \& editing. Miloudi GAZZAZ: Supervision, validation, \& review.

\section{Ethics and Reporting Guidelines}

Informed consent and verbal permission were obtained from the patient prior to the submission of this article. Also, this article respects both the Consensus-based Clinical Case Reporting Guideline and the Recommendations for the Conducting, Reporting, Editing, and Publication of Scholarly Work in Medical Journals [9] [10].

\section{References}

[1] Shiraishi, T., Crock, H.V. and Reynolds, A. (1995) Spinal Arachnoiditis Ossificans. European Spine Journal, 4, 60-63. https://doi.org/10.1007/BF00298421

[2] (2021) Arachnoiditis Ossificans. American Journal of Neuroradiology. http://www.ajnr.org/ajnr-case-collections-diagnosis/arachnoiditis-ossificans

[3] Lucchesi, A.C., et al. (1998) Review of Arachnoiditis Ossificans with a Case Report. https://www.barrowneuro.org/for-physicians-researchers/education/grand-rou nds-publications-media/barrow-quarterly/volume-14-no-4-1998/review-arachn oiditis-ossificans-case-report/

[4] Larry, B. (2007) Arachnoiditis. https://radsource.us/arachnoiditis/

[5] Frizzell, B., Kaplan, P., Dussault, R. and Sevick, R. (2001) Arachnoiditis Ossificans. American Journal of Roentgenology, 177, 461-464. https://doi.org/10.2214/ajr.177.2.1770461

[6] Bernard, P., Vanhoenacker, F. and Adam, N. (2010) Arachnoiditis Ossificans. Journal of the Belgian Society of Radiology, 93, 108. https://doi.org/10.5334/jbr-btr.363

[7] Chan, C., Lau, P., Sun, L. and Lo, S. (2009) Arachnoiditis Ossificans. Hong Kong Medical Journal, 15, 146-148.

[8] Steel, C.J., Abrames, E.L. and O’Brien, W.T. (2015) Arachnoiditis Ossificans-A Rare Cause of Progressive Myelopathy. Open Neuroimaging Journal, 9, 13-20. https://doi.org/10.2174/1874440001509010013

[9] Consensus-Based Clinical Case Reporting Guideline and the Recommendations for the Conducting, Reporting, Editing, and Publication of Scholarly Work in Medical Journals-Google Search [Internet].

https://www.google.com/search?q=Consensus-based+Clinical+Case+Reporting+Guid eline+and+the+Recommendations+for+the+Conducting\%2C+Reporting\%2C+Editin 
$\mathrm{g} \% 2 \mathrm{C}+$ and + Publication + of + Scholarly + Work + in + Medical + Journals\&oq=Consensu s-based+Clinical+Case+Reporting + Guideline + and + the + Recommendations + for + th e+Conducting $\% 2 \mathrm{C}+$ Reporting $\% 2 \mathrm{C}+$ Editing $\% 2 \mathrm{C}+$ and + Publication + of + Scholarly + Work + in + Medical + Journals\&aqs $=$ chrome..69i57.2034j0j15\&sourceid $=$ chrome\&ie $=$ $\underline{\mathrm{UTF}-8}$

[10] Gagnier, J.J., Kienle, G., Altman, D.G., Moher, D., Sox, H., Riley, D., et al. (2013) The CARE Guidelines: Consensus-Based Clinical Case Reporting Guideline Development. Global Advances in Health and Medicine, 53, 1541-1547.

https://doi.org/10.1111/head.12246 